\title{
Optimal real-space renormalisation
}

\author{
T A. Witten Jr and J J Prentis \\ Harrison M. Randall Laboratory of Physics, University of Michigan, Ann Arbor, Michigan \\ 48109, USA
}

Received 19 May 1980

\begin{abstract}
Using a continuous class of real-space renormalisation transformations we study the critical behaviour of the Ising model in a variety of two-dimensional lattices. We use the simplest possible cluster, with two cells, and a single renormalised coupling. Certain of these transformations-well separated from the majority spin rule-produce sharply more accurate critical properties than the rest. From this study we note an optimal set of conditions which characterise the successful transformations. The optimal transformations appear to have a certain 'locality' property: renormalised couplings beyond nearest neighbour are especially small.
\end{abstract}

\section{Introduction}

Matter near a second-order phase transition has a singular free energy per degree of freedom $f$ which has a well known scaling symmetry. Thus for an Ising ferromagnet at a temperature $t$ measured from the critical temperature and in a magnetic field $h$, the free energy $f$ satisfies the scaling law (Widom 1965, Fisher 1967)

$$
f(t, h)=\lambda^{-d} f\left(\lambda^{y_{t}} t, \lambda^{y_{h}} h\right)
$$

provided the fields $t$ and $h$ are sufficiently small. Here $d$ is the dimension of space, $\lambda$ is an arbitrary scale parameter and the exponents $y_{t}$ and $y_{h}$ determine the singular behaviour near the critical point. Recently, much progress has been made in understanding the scaling symmetry by use of the idea of renormalisation (Wilson and Kogut 1974, Niemeijer and van Leeuwen 1976, Brézin et al 1973). By renormalisation of an Ising model, we mean a mapping of each of its configurations onto a configuration of a similar system with fewer spins. If the distribution function of the renormalised system is characterised by the same Hamiltonian as the original system, one says that it is a fixed point. By postulating the existence of such fixed points, one may derive the scaling behaviour of equation (1) and the exponents $y_{t}$ and $y_{h}$. Typically, one determines the fixed point using some finite truncation of the real infinite system. Excellent results are often obtained even in severely truncated systems: the predicted critical indices $y_{t}$ and $y_{h}$ and the critical temperature come very close to their true values. The success of these approximations is the more puzzling in view of the formal pathologies of renormalisation (Griffiths and Pearce 1979). Further, the success of these approximations depends sensitively on how the truncation is made and on how the mapping into the renormalised spins is defined. The reasons for this sensitivity are not understood; the successful approximations must be found for each new system by trial and error. 
It is our purpose here to understand what makes a successful approximation and how the transformation may be optimised. Previous workers have studied the problem of optimisation. They wished to obtain good results for a particular case (Berker and Wortis 1976), or to satisfy some pre-specified extremal (Kadanoff 1975, Kadanoff et al 1976, Barber 1977, Shenker et al 1979) or self-consistency (Kadanoff and Houghton 1975. Subbarao 1975, Hsu and Gunton 1977, Lewis 1977, Mazenko et al 1978, Fiorenzato et al 1978) criterion. The resulting transformation often proves to be accurate in several respects. Our approach inverts the reasoning; we find accurate transformations empirically, and then look for criteria which characterise them. We study the Ising model in the simplest possible finite lattice approximation (Subbarao 1975, Niemeijer and van Leeuwen 1976)-two-cell clusters-with various boundary conditions. We allow for the most general mapping from the spins of a cell to the renormalised spin. As a consequence, there is a two-parameter family of renormalisation transformations with the popular majority spin rule as a special case. We vary these parameters to find the best transformation.

The results of this study are surprising and suggestive. We find that certain values of the parameters give sharply superior results. For one optimal type of truncation in each lattice there is a narrow range of the mapping parameters for which the exponents $y_{t}, y_{h}$ and the critical temperature are all as close as possible to their true values. The occurrence of this optimisation phenomenon is taken as evidence of the accuracy of our approximation, with the hope that there exist revealing criteria which determine these successful approximations. Indeed, we find that the cases which exhibit the optimisation property have certain features in common. They occur on periodically continued clusters rather than on those with free or mean-field boundaries. Where several periodic continuations are possible, only a subset show optimisation. The optimum point is successively better in the triangular, square and hexagonal lattices. The optimal parameters show a striking linear variation with the lattice coordination number. In a separate study we find that the optimal transformations appear to have especially small fixed-point couplings beyond the nearest-neighbour Ising coupling: the optimal transformation is 'local' at the fixed point.

In $\S 2$ we describe the parameterised real-space renormalisation transformation (RSRT). Section 3 describes the examples studied and displays the results of the calculations.

\section{Renormalisation transformation}

Our system is a set of $N$ Ising spins $\mu^{i}= \pm 1, i=1, \ldots, N$, on a two-dimensional lattice. The Hamiltonian $\mathscr{H}\{\mu\}$ contains a nearest-neighbour coupling and a magnetic field energy

$$
H\{\mu\} \equiv-\mathscr{H}\{\mu\} / k T=K \sum_{\langle i j\rangle} \mu^{i} \mu^{j}+h \sum_{i} \mu^{i} .
$$

The thermodynamics is determined by the prrtition function

$$
Z[H] \equiv \sum_{\{\mu\}} \exp (H\{\mu\})
$$

To define the renormalisation group transformation, we form cells of $b^{2}$ lattice sites, so that the lattice of cells has its lattice spacing increased by a scale factor $b$. Figure 1 shows 
(a)

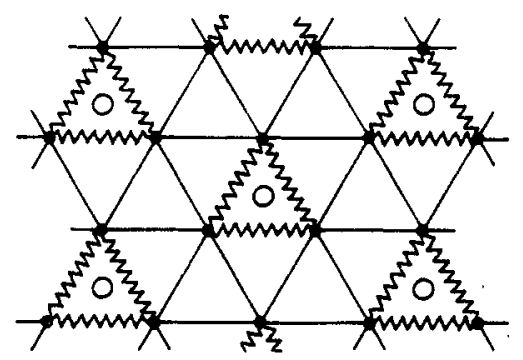

(b)

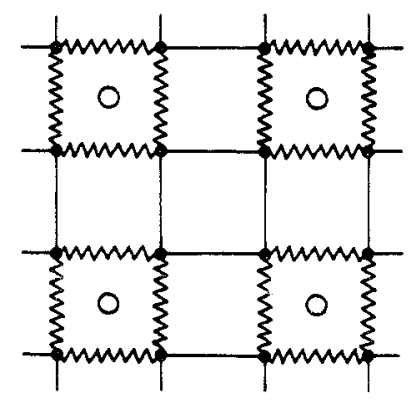

(c)

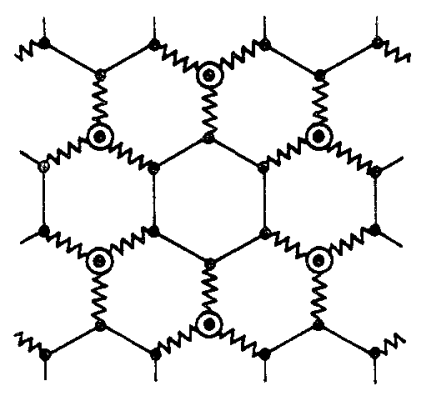

Figure 1. Formation of the cells in the renormalisation transformation on $(a)$ triangular, $(b)$ square, and (c) hexagonal lattices; $=$ site spin $\mu, O=$ cell spin $\mu^{\prime}$. The straight (jagged) lines represent the intercell (intracell) site-spin interactions.

how such cells may be constructed in the triangular $(b=\sqrt{3})$, square $(b=2)$ and hexagonal $(b=2)$ lattices. With each cell we associate a new Ising spin variable $\mu_{c}^{\prime}= \pm 1 ; c=1, \ldots, N^{\prime}=N / b^{2}$. The Hamiltonian of this cell-spin system is defined (see however Griffiths and Pearce 1979) by the renormalisation transformation

$$
\exp \left(H^{\prime}\left\{\mu^{\prime}\right\}\right) \equiv \sum_{\{\mu\}}\left(\prod_{c} P\left(\mu_{c}^{\prime} \mid \mu_{c}^{1} \ldots \mu_{c}^{4}\right)\right) \exp (H\{\mu\})
$$

where the weight factor (projection matrix) $P\left(\mu_{c}^{\prime} \mid \mu_{c}^{1} \ldots \mu_{c}^{4}\right)$ specifies the conditional probability that the given cell spin has the value $\mu_{c}^{\prime}$, given that its site spins have the values $\mu_{c}^{1} \ldots \mu_{c}^{4}$ (in the triangular-lattice cell $\mu_{c}^{4}$ is absent). We shall consider a class of projections $P$ restricted as follows:

(i) The transformation must preserve the partition function: $Z\left[H^{\prime}\right]=Z[H]$. This restriction assures that the scaling behaviour of equation (1) will emerge near the critical point (Niemeijer and van Leeuwen 1976). The partition function is preserved if the 
projection matrix $P$ is properly normalised, that is

$$
\sum_{\mu_{c}^{\prime}} P\left(\mu_{c}^{\prime} \mid \mu_{c}^{1} \ldots \mu_{c}^{4}\right)=1
$$

for arbitrary fixed $\mu_{c}^{1} \ldots \mu_{c}^{4}$.

(ii) The transformation must preserve spin-reversal symmetry: $P\left(\mu^{\prime} \mid \mu\right)=$ $P\left(-\mu^{\prime} \mid-\mu\right)$. This assures that $H^{\prime}$ is even under spin reversal whenever $H$ is. Thus the fixed-point Hamiltonian corresponding to the Ising critical point will be even under spin reversal.

(iii) The projection should be a symmetric function of the $\mu^{i}$ spins, since all the $\mu^{i}$ spins are equivalent.

(iv) We shall not require that $P$ be non-negative.

The most general projection matrix satisfying the requirements (i)-(iii) may be written

$$
P\left(\mu_{c}^{\prime} \mid \mu_{c}^{1} \ldots \mu_{c}^{4}\right)= \begin{cases}w, & \mu_{c}^{1}=\mu_{c}^{2}=\mu_{c}^{3}=\mu_{c}^{4}=\mu_{c}^{\prime} \\ 1-w, & \mu_{c}^{1}=\mu_{c}^{2}=\mu_{c}^{3}=\mu_{c}^{4}=-\mu_{c}^{\prime} \\ v, & \text { exactly one of } \mu_{c}^{1} \ldots \mu_{c}^{4}=-\mu_{c}^{\prime} \\ 1-v, & \text { exactly one of } \mu_{c}^{1} \ldots \mu_{c}^{4}=\mu_{c}^{\prime} \\ 0 \cdot 5, & \text { otherwise. }\end{cases}
$$

For the triangular lattice $\mu_{c}^{4}$ is absent.

Having defined the projection matrix $P$, we consider a truncated lattice on which the renormalised Hamiltonian $H^{\prime}$ may be (approximately) calculated. We choose the simplest truncation scheme possible: a two-cell cluster. The only terms possible in $H^{\prime}$ are then those of the original Hamiltonian (equation (2)), plus a constant $K_{0}^{\prime}$. The renormalisation specifies the couplings $K_{0}^{\prime}, K^{\prime}$, and $h^{\prime}$ of this Hamiltonian as a function of the original $K$ and $h$.

We form two-cell clusters in several ways, which differ in how the boundary is treated (figure 2). In a free cluster there are no couplings except within the cluster. As compared with the infinite lattice, the free cluster underestimates the energy of most configurations. A second choice of boundary condition is the mean-field boundary (Myles and Dow 1979), in which the cluster is presumed to be surrounded with spins equal in magnitude to the average of the cluster spins in that configuration. A third choice is to continue the two-cell cluster periodically. This scheme preserves the translational invariance of the Hamiltonian; for every state of a given energy, any translation of the system yields a state of the same energy. But with periodic boundaries the energy per spin is overestimated as compared with the infinite system at the same temperature. For the square lattice two periodic continuations are possible. The $\mathrm{A}$ and $B$ cells may be arranged either in rows or in a checkerboard pattern.

Typically, the transformation defined on these clusters has a 'fixed point' $K^{*}$, where $K^{\prime}=K=K^{*}, h^{\prime}=h=0$, and the transformation is approximately linear for $K \approx K^{*}$, $h \approx 0$ :

$$
K^{\prime}-K^{*}=b^{y_{t}}\left(K-K^{*}\right), \quad h^{\prime}=b^{y_{h}} h
$$

for certain values of $y_{t}$ and $y_{h}$. If the same transformation $(h, K) \rightarrow\left(h^{\prime}, K^{\prime}\right)$ were to apply in the infinite, untruncated system, it would imply (Niemeijer and van Leeuwen 1976) the scaling behaviour of equation (1). In the next section we study the way in which $w$ and $v$ affect our approximate values of $K^{*}, y_{h}$, and $y_{t}$. 

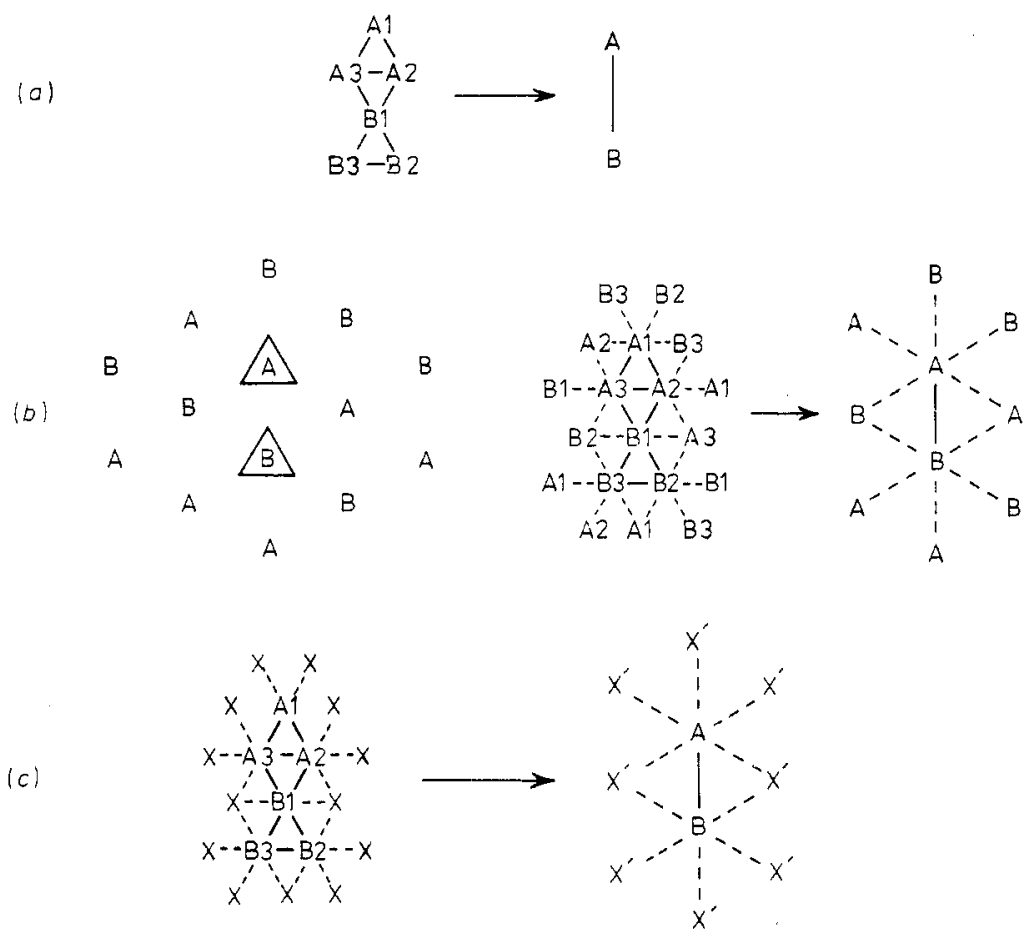

Figure 2. Boundary conditions used for the triangular lattice two-cell cluster, (a) Free cell for the site spins (left picture) and the cell spins (right picture); sites $\mathrm{A} 1, \ldots, \mathrm{B} 3$ are positions of spins $\mu_{\mathrm{A}}^{1}, \ldots, \mu_{\mathrm{B}}^{3}$. Lines between sites denote an Ising coupling $K \mu \mu$ between the corresponding spins. (b) Periodic boundary: Left-hand picture shows the periodic continuation of the A-B cluster. Middle picture shows one cluster with its boundary sites. Dashed lines denote $\frac{1}{2} K \mu \mu$ couplings (each of which would be counted twice in the continued lattice). Right-hand picture shows the corresponding cluster of cell spins. (c) Mean-field boundary: sites $X$ have a spin equal to $\frac{1}{6}\left(\mu_{A}^{1}+\ldots+\mu_{B}^{3}\right)$. Sites $X^{\prime}$ have a spin equal to $\frac{1}{2}\left(\mu_{\mathrm{A}}^{\prime}+\mu_{\mathrm{B}}^{\prime}\right)$

\section{Results}

A striking feature of our results is the importance of the parameter $w$ which controls the projection of the unanimous states. When $w$ is one, a ferromagnetic ground state will be mapped completely into another. We find that $w-1$ must be less than $\sim 0 \cdot 01$ to preserve the correct qualitative fixed point behaviour. This great sensitivity to $w$ arises numerically from the large Boltzmann weight of the ground state compared with other states of the truncated system. In the results reported below we have taken $w=1$, so the unanimous states are preserved identically.

The parameter $v$ has a more complicated effect on the fixed-point behaviour. We recall that $v$ controls the mapping of the next-to-unanimous state of a cell, and that $\dot{v}=w=1$ corresponds to the majority spin rule. Figure 3 summarises the effects of $v$ by displaying $K^{*}, y_{t}$, and $y_{h}$ as functions of $v$ for each truncated lattice we have studied. In most of these truncations there is no $v$ which is distinctly 'best'. The $v$ which brings $K^{*}$ closest to its true value is far from the $v$ values which bring $y_{t}$ or $y_{h}$ closest to their true values. Figures $3(b),(f)$, and $(h)$ show a striking difference: the $v$ values which optimise $K^{*}, y_{t}$, and $y_{h}$ differ by a percent or less: there is an optimal $v$. 

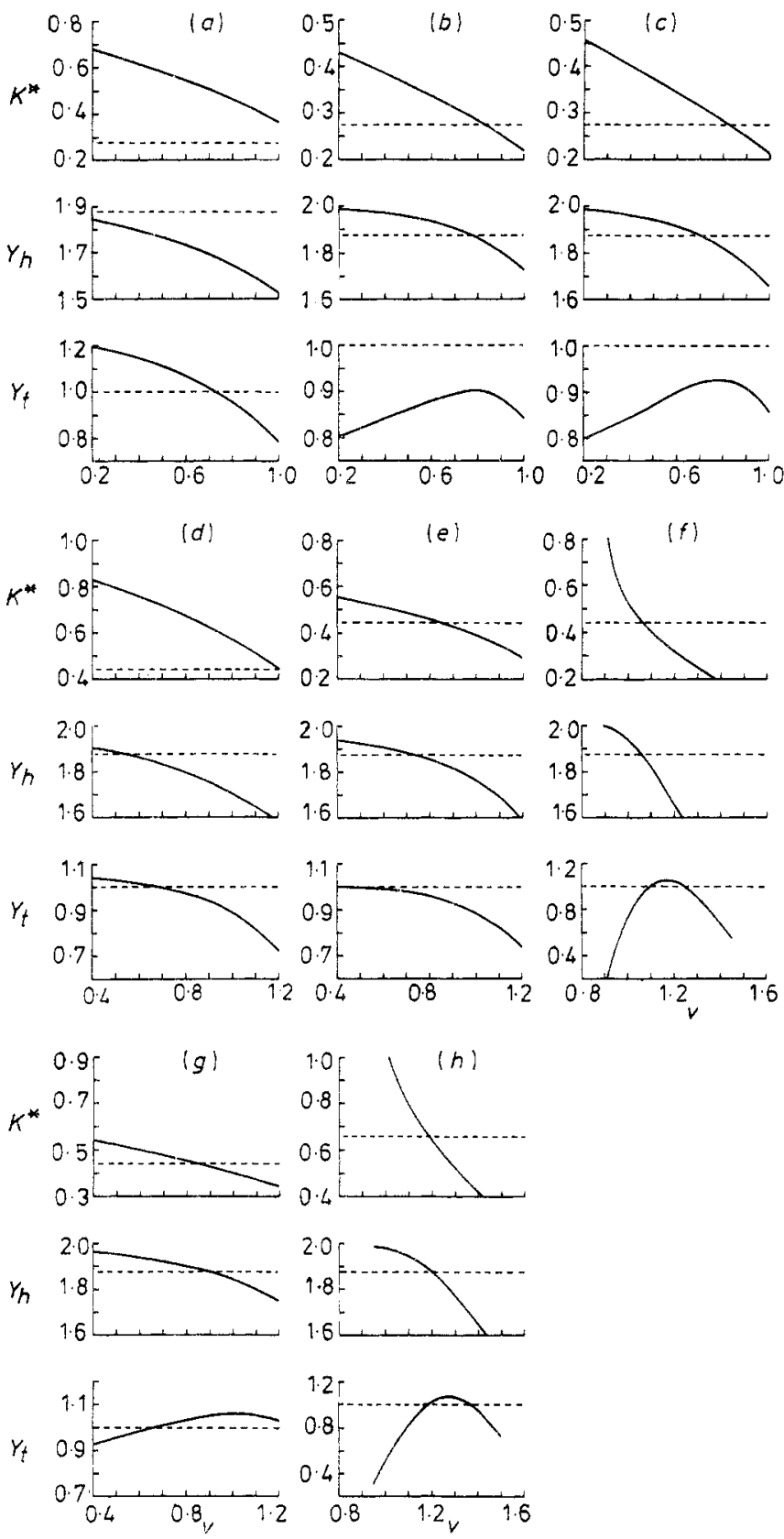

Figure 3. The critical indices $K^{*}, y_{\mathrm{h}}$ and $y_{\mathrm{t}}$ calculated using the two-cell cluster renormalisation transformation as a function of the projection parameter $v(w=1)$. The horizontal dashed lines indicate the exact known values at the Ising critical point. Triangular lattice: ( $a$ ) free boundary; (b) periodic boundary; (c) mean-field boundary. Square lattice: $(d)$ free boundary; $(e)$ periodic boundary (row continuation); $(f)$ periodic boundary (checkerboard continuation); $(g)$ mean-field boundary. Hexagonal lattice: $(h)$ periodic boundary. 
The three cases which show this behaviour are virtually all $†$ periodic continuations-one for each lattice type. But not all periodic continuations show this behaviour (cf figure $3(e)$ ). There is a nearly linear relationship between the optimal $v$ values for the three lattices (table 1 , left column) and their coordination number $n$. A linear extrapolation based on these values from the square and hexagonal lattices predicts $n=6.03$ for the triangular lattice-a half percent from its true value. We note further that $y_{t}$ appears to attain a maximum near the optimal $v . \ddagger$

These results suggest that the optimal $v$ minimises the sources of error in our approximate RSRT. A major source of error is our neglect of couplings beyond the nearest-neighbour coupling. We expect that these further couplings would be small if
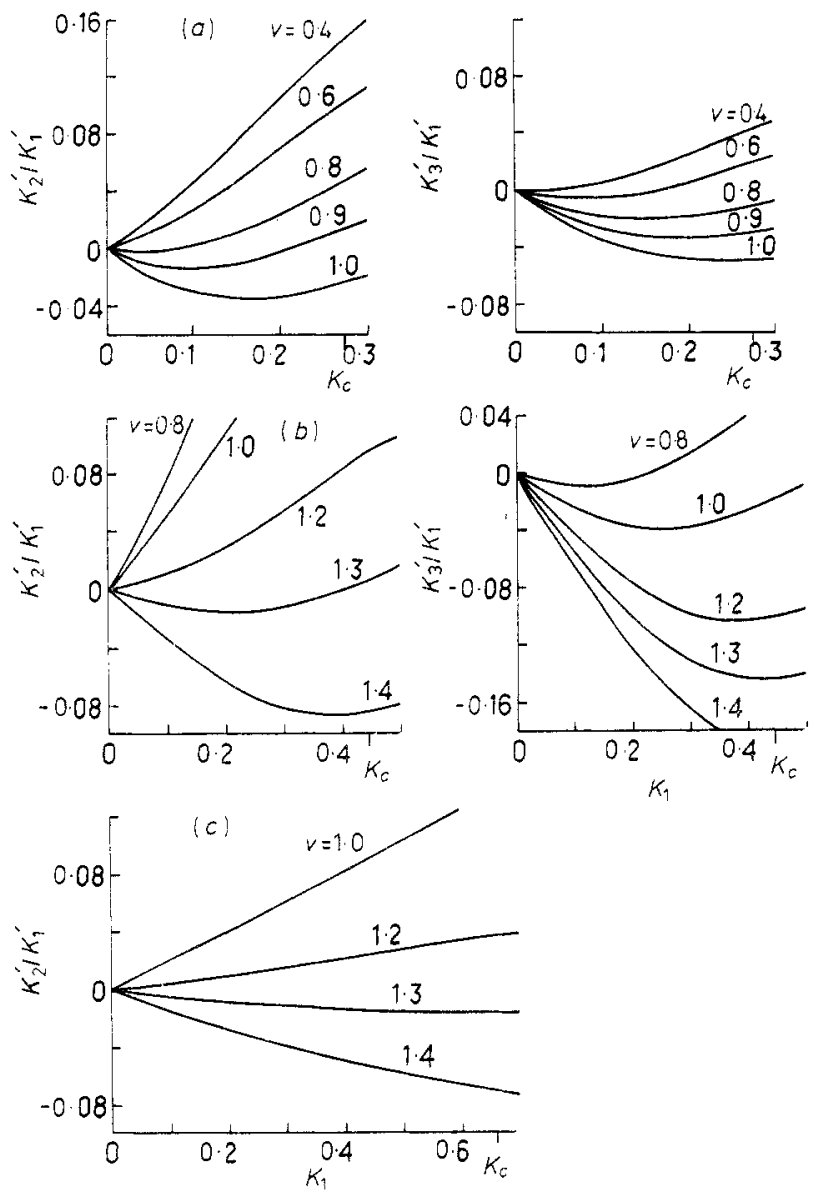

Figure 4. Second $\left(K_{2}^{\prime}\right)$ and third $\left(K_{3}^{\prime}\right)$ nearest-neighbour couplings (relative to $K_{1}^{\prime}$ ) calculated in the second-order cumulant approximation as a function of the nearestneighbour coupling $K_{1}$ and projection parameter $v(w=1)$. (a) Triangular lattice. (b) Square lattice. (c) Hexagonal lattice.

+ The 'mean-field' case of figure $3(c)$ shows optimisation comparable with the periodic case of figure $3(b)$. Indeed, these two transformations are so similar in all respects that they can hardly be considered as independent.

$\$$ That exponents should be insensitive to the renormalisation parameters (as at the extremal point) was suggested by Bell and Wilson (1975). 
an exact renormalisation were done with our optimal projection. To check this idea, we made a separate study of these further couplings in the cumulant approximation (Niemeijer and van Leeuwen 1974). This study confirmed the idea that the further couplings are small at the optimum point.

In the second-order cumulant expansion one obtains second- and third-neighbour couplings $K_{2}^{\prime}$ and $K_{3}^{\prime}$ in addition to the Ising coupling $K_{1}^{\prime}$, if the unprimed coupling is purely Ising. The formulae for these are given in the Appendix. We studied the behaviour of $K_{2}^{\prime}$ and $K_{3}^{\prime}$ as a function of $K_{1}$ and $v$. Figure 4 shows the results for triangular, square, and hexagonal lattices. For each lattice there is a small range of $v$ values that makes $K_{2}^{\prime}$ and $K_{3}^{\prime}$ both small for $0<K_{1} \leqslant K_{c}$. Within this range one may find a $K_{1}^{*}$ by neglecting $K_{2}^{\prime}$ and $K_{3}^{\prime}$. If one adjusts $v$ so that $K_{1}^{*}=K_{c}$, then $K_{2}^{\prime}$ and $K_{3}^{\prime}$ are small and of opposite sign (figure 5). The results for the hexagonal lattice are particularly striking. Here only $K_{2}^{\prime}$ is non-zero in our second-order treatment. By examining the expressions for $K_{1}^{\prime}$ and $K_{2}^{\prime}$ one readily shows that any $w$ and $v$ which make $K_{2}^{\prime}=0$ at $K_{1}^{*}$ also make $K_{1}^{*}$ equal to the exact $K_{c}=\frac{1}{2} \ln (2+\sqrt{3})$. There is a rough agreement between the best $v$ values found in this cumulant study and those found on the truncated lattices, as table 1 shows.
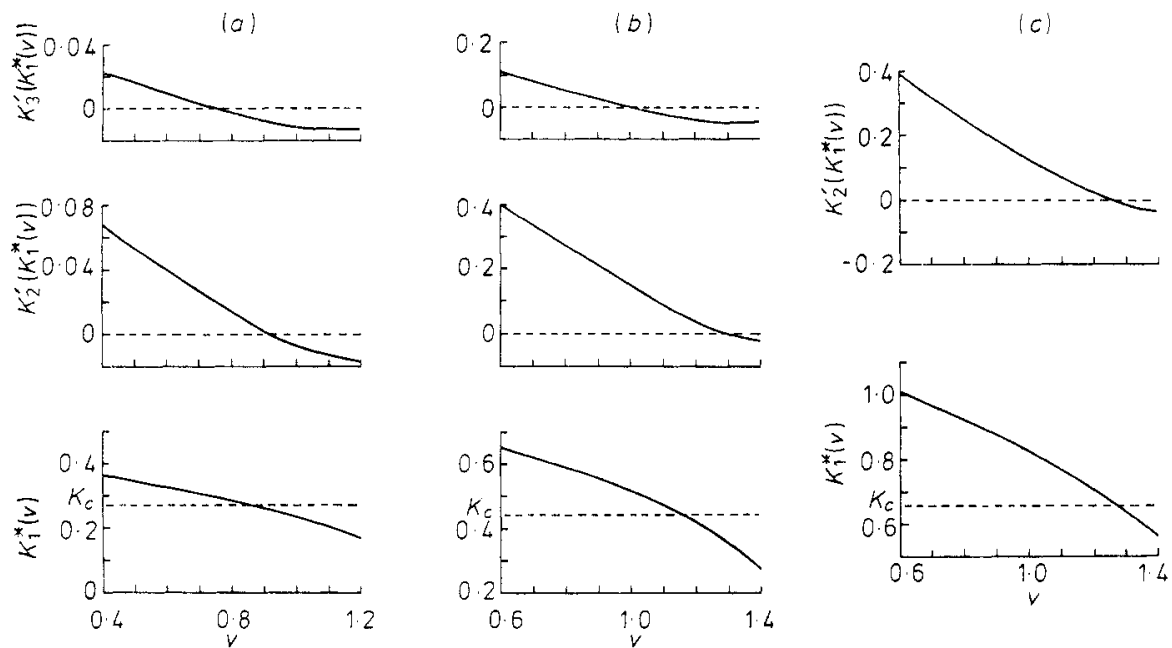

Figure 5. The nearest-neighbour fixed point coupling $K_{1}^{*}$ (neglecting $K_{2}^{\prime}$ and $K_{3}^{\prime}$ ) calculated in the second-order cumulant approximation as a function of the projection parameter $v(w=1)$. Also shown are the values of the further couplings $K_{2}^{\prime}$ and $K_{3}^{\prime}$ at this fixed point. (a) Triangular lattice. (b) Square lattice. (c) Hexagonal lattice.

Table 1. The values of the projection parameter $v$ which make $K^{*}=K_{c}$ in our two-cell cluster and the cumulant approximations.

\begin{tabular}{lll}
\hline Lattice & $v$ (Two-cell cluster) & $v$ (Cumulant) \\
\hline Triangular & $0 \cdot 8197$ (figure $3(b))$ & $0 \cdot 8502$ (figure $5(a)$ ) \\
Square & $1 \cdot 0645$ (figure $3(f)$ ) & $1 \cdot 1642$ (figure $5(b)$ ) \\
Hexagonal & $1 \cdot 1854$ (figure $3(h))$ & $1 \cdot 2685$ (figure $5(c)$ ) \\
\hline
\end{tabular}




\section{Discussion}

We wish to explain the striking agreement between the true critical behaviour and that of our optimal RSRT's. One possible explanation is that a specific choice of RSRT happens to produce compensating errors in an intrinsically bad approximation, so that, for instance $K^{*}$ comes out correctly. But then one must explain the gratuitous agreement of the two other quantities - the two critical exponents-for the same choice of transformation. The same phenomenon of 'automatic agreement' has been a common occurrence in other RSRT studies (Kadanoff and Houghton 1975, Kadanoff 1975).

A second explanation appears more plausible; that the optimal RSRT's have especially small errors. That is, the approximate fixed-point behaviour we have found with our optimal transformations is especially close to the behaviour under these same transformations with no approximations. The most obvious approximation we have made is our neglect of all couplings beyond nearest-neighbour Ising coupling in the fixed-point Hamiltonian. Thus we would expect the exact RSRT to yield a 'local' fixed point close to the Ising critical point, with only small couplings beyond nearestneighbour. Our study of these further couplings in the cumulant approximation confirms this idea. It shows that for the optimal RSRT, the further couplings studied are especially small. This suggests a method of finding optimal RSRT's in future work: that is to choose the RSRT so as to minimise the further couplings.

Again, several features of the optimum RSRT's remain to be explained. It is not clear why a single choice of transformation tends to make more than one further coupling small at the same time. These findings suggest that there is some important feature of real-space renormalisation waiting to be discovered. We believe that the technique of varying the transformation as applied here can lead to an understanding of this undiscovered feature. Then one may replace trial-and-error searching by predictions of when a given transformation will be successful for a particular problem.

\section{Acknowledgment}

It is a pleasure to thank Professor Michael Wortis for stimulating discussions and criticism. Messrs A N Berker and L Kadanoff provided helpful information.

\section{Appendix. Renormalisation transformations in the cumulant approximation (with general projection)}

In order to compute the transformation, we require the distribution function for the $\mu^{\prime}$ spins for a given nearest-neighbour coupling $K$ between the unprimed spins $\mu$. We treat the intracell couplings exactly and the intercell couplings to second order in $K$ using the cells of figure 1 . The primed couplings thus generated may be expressed in terms of a 'susceptibility' and a correlation function for the isolated cell. We define the susceptibility $\chi_{i}$ by $\left\langle\mu^{i}\right\rangle_{0} \equiv \chi_{i} \mu^{\prime}$ ', and the correlation $\Gamma_{i j}$ by $\left\langle\mu^{i} \mu^{j}\right\rangle_{0} \equiv \Gamma_{i j}$. Here

$$
\langle A\rangle_{0} \equiv \frac{\Sigma_{\mu^{1} \ldots \mu^{4}} A\left(\mu^{1} \ldots \mu^{4}\right) P\left(\mu^{\prime} \mid \mu^{1} \ldots \mu^{4}\right) \exp \left(H_{0}\left(\mu^{1} \ldots \mu^{4}\right)\right)}{\Sigma_{\mu^{1} \ldots \mu^{4}} P\left(\mu^{1} \mid \mu^{1} \ldots \mu^{4}\right) \exp \left(H_{0}\left(\mu^{1} \ldots \mu^{4}\right)\right)}
$$

and $H_{0}$ is the Hamiltonian of the isolated cell. 
For the triangular lattice the unperturbed cell partition function $z_{0}$ is given by $z_{0}=\exp (3 K)+3 \exp (-K)$. The correlations $\Gamma_{i j}$ are all equal and independent of the projection parameters $v$ and $w$ :

$$
\Gamma_{i j} \equiv \Gamma=(\exp (3 K)-\exp (-K)) / z_{0}
$$

The susceptibility $\chi_{i}$ has the form

$$
\chi_{i} \equiv \chi=[(2 w-1) \exp (3 K)+(2 v-1) \exp (-K)] / z_{0} .
$$

To second order in $K$, only nearest-, second- and third-neighbour couplings $K_{1}^{\prime}, K_{2}^{\prime}$ and $K_{3}^{\prime}$ are induced:

$$
\begin{aligned}
& K_{1}^{\prime}(K)=2 \chi^{2} K+4 \chi^{2}\left(1+\Gamma-2 \chi^{2}\right) K^{2}, \\
& K_{2}^{\prime}(K)=\chi^{2}\left(1+7 \Gamma-8 \chi^{2}\right) K^{2}, \\
& K_{3}^{\prime}(K)=4 \chi^{2}\left(\Gamma-\chi^{2}\right) K^{2} .
\end{aligned}
$$

For the square lattice $z_{0}=\exp (4 K)+\exp (-4 K)+6$. Two correlations enter:

$$
\Gamma_{12}=(\exp (4 K)-\exp (-4 K)) / z_{0}
$$

for adjacent sites, and

$$
\Gamma_{13}=(\exp (4 K)+\exp (-4 K)-2) / z_{0}
$$

for opposite sites. The susceptibility $\chi$ is

$$
\chi=[(2 w-1) \exp (4 K)+4 v-2] / z_{0} .
$$

The nearest-neighbour coupling is found to have no second-order part:

$$
K_{1}^{\prime}(K)=2 \chi^{2} K
$$

The others are

$$
\begin{aligned}
& K_{2}^{\prime}(K)=2 \chi^{2}\left(1+2 \Gamma_{12}+\Gamma_{13}-4 \chi^{2}\right) K^{2}, \\
& K_{3}^{\prime}(K)=2 \chi^{2}\left(\Gamma_{12}+\Gamma_{13}-2 \chi^{2}\right) K^{2} .
\end{aligned}
$$

In the hexagonal lattice $z_{0}=\exp (3 K)+\exp (-3 K)+3 \exp (K)+3 \exp (-K)$. The correlations $\Gamma_{12}$ of the outer spins are

$$
\Gamma_{12}=(\exp (3 K)+\exp (-3 K)-\exp (K)-\exp (-K)) / z_{0}
$$

Their susceptibilities are

$$
\chi_{1}=\left[(2 w-1) \exp (3 K)+(2 v-1)(\exp (-3 K)+\exp (K)] / z_{0}\right.
$$

Only nearest and second couplings are induced:

$$
\begin{aligned}
& K_{1}^{\prime}(K)=2 \chi_{1}^{2} K \\
& K_{2}^{\prime}(K)=\chi_{1}^{2}\left(1+3 \Gamma_{12}-4 \chi_{1}^{2}\right) K^{2} .
\end{aligned}
$$

\section{References}


Brézin E, Le Guillou J C and Zinn-Justin J 1973 Phys. Rev. D8 434, 2418

Fiorenzato A, Stella A L and Toigo F 1978 Phys. Lett. 69A 127

Fisher M E 1967 Rep. Prog. Phys. 30615

Griffiths R B and Pearce P A 1979 J. Statist. Phys. 20499

Hsu S-C and Gunton J D 1977 Phys. Rev. B 152688

Kadanoff L P 1975 Phys. Rev. Lett. 341005

Kadanoff L P and Houghton A 1975 Phys. Rev. B 11377

Kadanoff L P, Houghton A and Yalabik M C 1976 J. Statist. Phys. 14171

Lewis A L 1977 Phys. Rev. B 161249

Mazenko G F, Nolan M J and Valls O T 1978 Phys. Rev. Lett. 41500

Myles C W and Dow J D 1979 Phys. Rev. B 194939

Niemeijer Th and van Leeuwen J M J 1974 Physica 7117

- 1976 Phase Transitions and Critical Phenomena vol 6, eds. C Domb and MS Green (London: Academic)

Shenker S J, Kadanoff L P and Pruisken A M M 1979 J. Phys. A: Math. Gen. 1291

Subbarao K 1975 Phys. Rev. B 111165

Widom B 1965 J. Chem. Phys. 433898

Wilson K G and Kogut J 1974 Phys. Rep. 12C 76 